\title{
Crítica, emancipación y educación
}

\section{Margarita R. Sgró}

Professora da Universidad Nacional del Centro de la Provincia de Buenos Aires

\section{Resumen}

Conciliar las figuras del intelectual y del maestro no siempre es posible. La alquimia entre quien produce saber y quien apasionadamente lo transmite es, a veces, conflictiva por las demandas que tiene una y otra actividad, sin embargo el Prof. Pedro Goergen reúne ambas condiciones, quienes fuimos sus alumnos podemos dar fe de ello. El presente artículo analiza las relaciones entre crítica, emancipación y educación que aparecen en la obra de Goergen, resaltando el permanente llamado de atención contra la deshumanización creciente de nuestra civilización manifiesta en una "técnica" autonomizada y la esperanza en una educación crítica capaz de interrumpir ese camino hacia la autodestrucción.

Palabras Clave: Educación; Emancipación; Crítica.

\begin{abstract}
Reconciling the figures of the intellectual and that of the teacher is not always possible. The alchemy between the one who produces knowledge and the one who passionately communicates it is sometimes conflicting because of the demands that each activity has. However, Professor Pedro Goergen meets both conditions. We, who were his students, may attest to that. The present article analyzes the relations between critique, emancipation and education that come up in Goergen's work, highlighting his permanent call of attention against the growing dehumanization of our civilization that manifests on an empowering 'technique' and the hope of a critical education capable of interrupting the journey towards self-destruction.
\end{abstract}

Keywords: Education; Emancipation; Critique.

Filosofia e Educação [RFE] - Volume 8, Número 2 - Campinas, SP

Junho-Setembro de 2016 - ISSN 1984-9605 - p. 79-96 


\section{Primeras palabras}

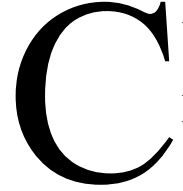

uando decidimos dedicarle un libro al Profesor Pedro Goergen pensé que un texto que homenajee a nuestro Orientador de doctorado podía reunir dos figuras, una la del Maestro, de aquel que guía, orienta, abre otras perspectivas de análisis. Otra figura la del Intelectual, ambas, se conjugan en la persona del Prof. Pedro. Si hablara de él como maestro lo caracterizaría como alguien generoso en la enseñanza, disimulando la pose tradicional del que sabe, divertido, incisivo en sus apreciaciones, preguntando para sembrar dudas y dejándonos más preocupados y menos omnipotentes después de cada entrevista. Reuniones, que en mi caso particular eran también largas charlas sobre la Argentina, su suerte política que por aquellos años 1999-2004 era una tragedia y comparaciones con Brasil que me permitieron aprender cómo dos países tan próximos podían tener una cultura tan diferente, valoro mucho esas "conversas" porque me ayudaron a no extrañar tanto mis charlas con compañeros de militancia política en Tandil, mi ciudad natal. Recuerdo como tratábamos de desentrañar, sin comprender mucho el fenómeno Hugo Chavez, que en Venezuela mezclaba el militarismo y la espada de Bolívar con Gramsci y Eva Perón, entre otros. El Peronismo, Menem, Duhalde y De la Rúa presidentes argentinos de triste memoria, también eran temas de conversación, junto a la estrella ascendente del PT, Lula y la cultura política brasileña.

Si menciono con bastante detalle estos temas es porque ayudaron mucho a comprender y a querer un país y un pueblo al que admiro y del cual desde el primer día me sentí parte.

El Profesor Pedro impulsó además la formación de un grupo de estudios entre sus orientados denominado Grupo de Estudios en Filosofía,

Filosofia e Educação [RFE] - Volume 8, Número 2 - Campinas, SP Junho-Setembro de 2016 - ISSN 1984-9605 - p. 79-96 
Educación y Modernidad, que se ha mantenido emprendiendo diferentes actividades conjuntas hasta la actualidad lo que queda patentizado en el presente libro.

Para la institución de la cual provengo, la Facultad de Ciencias Humanas de la Universidad Nacional del Centro de la Provincia de Buenos Aires, con sede en la ciudad de Tandil-Buenos Aires-Argentina, el Profesor es alguien de consulta permanente, miembro del Consejo Asesor del Posgrado en Educación y uno de los impulsores de la apertura del Programa de Posgrado hace ya 18 años. Tiempo en el que hemos contado de manera incondicional con su colaboración. Por eso vaya en estas líneas mi reconocimiento personal y el de la Institución en la que me formé y trabajo.

El desarrollo de la filosofía de la educación en Brasil es muy importante comparado con Argentina. Hay profundidad y solidez en los debates que se generan justamente porque autores y corrientes de pensamiento se estudian con rigurosidad, constituyendo marcos de referencia a partir de los cuales pueden encuadrarse los fenómenos sociales y educacionales en su complejidad. Por contrapartida los estudios más frecuentes en el campo educacional han roto con la referencia a un marco teórico que se asuma en toda su dimensión, es decir admitiendo también las consecuencias. Dicho de otro modo, la mayor parte de los estudios educacionales carecen de la relación con una teoría social que encuadre el problema educacional y permita un debate más amplio y sustancial que aquél que surge simplemente de miradas recortadas sobre objetos extremadamente parcializados y analizados con una pretensión descriptiva que casi nunca se corrobora. Esta limitación, que en Argentina es muy visible, es una preocupación que no aparece en general ni en los cursos de educación de las universidades y mucho menos aún de la formación de profesores que se realiza fuera de las universidades en Institutos de 
formación docente especialmente creados para ello. La investigación en educación abandonó mayoritariamente las cuestiones relacionadas a la fundamentación y a los criterios de la crítica. Estas problemáticas, que raramente son debatidas en los cursos de ciencias de la educación, fueron presentadas en diversos espacios de debates académicos cuyos ejes residían en la fundamentación de la educación, un tema que fue obturado por la asunción tácita de que buscar fundamentos o justificaciones significaba, mantenerse nostálgicamente en discusiones superadas. Mientras en Argentina sólo algunos contados intelectuales persistían por fuera de la avalancha tecnicista, en Unicamp, pero no sólo en ella, también en otras universidades brasileñas había un campo, el de la filosofía de la educación, que posibilitaba una reflexión más compleja, más amplia y más comprehensiva. En ese sentido, muchos de nosotros fuimos profundizando una lectura sistemática de la Teoría crítica de la sociedad, guiados por un “orientador" y un grupo de "orientados" que sistemáticamente también, nos reuníamos para discutir los proyectos de investigación y los avances que íbamos realizando. Recuerdo esas reuniones como parte de una de las experiencias más ricas de aprendizaje que haya tenido y que siempre intenté replicar, en lo posible, con mis grupos de trabajo.

Vayan estas primeras palabras para resumir en una persona la conjunción de un maestro preocupado con la enseñanza y de un intelectual convencido de que la producción de saberes requiere de estudio riguroso pero también de discusiones y críticas que ayudan a "hilar más fino", a argumentar para defender posiciones teóricas y comprender las de los otros. Si tuviese que definir el aprendizaje académico más importante de mi formación en Unicamp diría que fue la comprobación de que es imprescindible reconstruir el campo de la Teoría de educación, si queremos pensar e incidir responsablemente en los rumbos de una sociedad que, como

Filosofia e Educação [RFE] - Volume 8, Número 2 - Campinas, SP Junho-Setembro de 2016 - ISSN 1984-9605 - p. 79-96 
ha dicho reiteradamente Goergen, precisa humanizarse con urgencia. En ese proceso de humanización la educación tiene una responsabilidad que debe ser pensada.

\section{La compleja responsabilidad de la educación}

Estamos saindo da década do "já não há nada a fazer". Nosso destino individual e social era governado pelas leis ocultas e poderosas do mercado [...]. A desfragmentação entre o individual e o coletivo era quase completa: não havia mais projeto individual nem coletivo de emancipação. [...] A sensação era de desamparo e desorientação, sentimentos anestesiados pela alucinada busca do aqui e agora.

Se falar dessas coisas como se elas pertencessem ao passado é extemporâneo porque elas ainda não mudaram, faço-o mesmo assim como forma de arriscar o diagnóstico de que nos encontramos no limiar do surgimento de uma nova consciência. [...] uma consciência de que é urgente e também possível fazer algo para curar as feridas da profunda degradação em que a sociedade fecha o segundo milênio. (GOERGEN, 2001, p. 6)

El autor de estas líneas elige correr el riesgo de hablar en tiempo pasado de las calamidades que caracterizan a la sociedad occidental, no lo hace por ingenuidad ni por simple necesidad de enunciar algunas frases retóricas que justifiquen la apuesta por una sociedad más humana, sino más bien para ubicar en ese contexto un análisis crítico que, al mismo tiempo, que sea impiadoso con las catástrofes del presente devele la necesidad de luchar contra ellas marcando el rumbo de la emancipación social.

Filosofia e Educação [RFE] - Volume 8, Número 2 - Campinas, SP Junho-Setembro de 2016 - ISSN 1984-9605 - p. 79-96 
Para un intelectual iluminista la necesidad y la posibilidad de luchar por la emancipación social no pueden solamente enunciarse como expresión de deseos, deben ser racionalmente justificadas. Ese esfuerzo puede emprenderse a partir de una lectura dedicada que permita ir armando y actualizando, los conceptos de teoría social que posibiliten estudiar la educación más allá del aula o de la escuela como institución. Cualquier teoría que pretenda atribuirse el calificativo de "crítica" debe pensar lo educativo en primer término en su dimensión ético-política y emancipatoria. Esta es una de las líneas de pensamiento más exploradas en la obra del Prof. Pedro Goergen.

A educação deve dar-se a partir da realidade sociocultural e econômica, [...] mas isso não pode ocorrer pelo fomento de uma atitude de condescendência com os traços desumanos, injustos, destrutivos e antiéticos que esta realidade ostenta. $\mathrm{O}$ preço que a escola paga pela submissão acrítica aos ditames da razão instrumental é a perda da dimensão ético-política do projeto histórico de emancipação. (GOERGEN, 2001, p. 83)

La relación entre crítica, emancipación y educación es una relación que se torna compleja por la doble responsabilidad que asumió la educación en las sociedades modernas, por un lado está encargada de reproducirlas simbólicamente y por otro de innovarlas.

En mucha de la producción teórica, la innovación social se entiende muy frecuentemente como una forma de acompañar la dirección moral e intelectual que las sociedades siguen, a veces innovar, es también modernizar la sociedad y en ella la escuela bajo la forma de incorporación de tecnología, las Tecnologías de información y comunicación (TICs) están 
ocupando un lugar cada vez más importante en las ciencias de la educación, se las estudia desde una perspectiva instrumental y a menudo se deposita en ellas demasiadas esperanzas de transformación. En ese sentido, como dice Goergen, la escuela no puede desentenderse del contexto cultural pero no tiene porque ser "condescendiente" con él, no cuestionando los problemas de desigualdad e injusticia que aquejan al mundo contemporáneo.

A ciência e a tecnologia, os dois fogosos cavalos de batalha do iluminismo, conduziram a carruagem do mundo ocidental, a par dos lugares de conforto e bem-estar, à beira dos abismos assustadores das dicotomias individuais e sociais em que segurança e fragilidade, conhecimento e ignorância, riqueza e pobreza, saúde e doença, opulência e miséria, vida e morte, coabitam lado a lado. (GOERGEN, 2010, p. 6)

La teoría crítica de educación no puede desconocer este panorama de injusticias en las que se debate el mundo contemporáneo y por ello recolocar el tema de la crítica y la emancipación social es más que un recurso para seguir produciendo conocimiento, es una obligación para todos aquellos que aún pensamos que es posible torcer el rumbo de un sistema que desampara sistemáticamente a millones de personas.

Dicho esto, debemos reconocer que para analizar la relación entre crítica, emancipación y educación es necesario colocar la discusión sobre el propio criterio de la crítica. Es decir, reflexionar sobre el concepto de crítica, sus alcances y sus limitaciones en el escenario de las sociedades contemporáneas caracterizadas por la diversidad y la complejidad.

Aparentemente todas as tentativas de caracterizar as mudanças que vivemos como um período pós (moderno) fracassaram. Dois aspectos

Filosofia e Educação [RFE] - Volume 8, Número 2 - Campinas, SP Junho-Setembro de 2016 - ISSN 1984-9605 - p. 79-96 
parecem destacar-se nas mudanças que hoje ocorrem: de um lado, a sociedade industrial se retira silenciosa como pela porta dos fundos, pressionada pelos riscos que trouxe, portanto, sem nenhum estrondo revolucionário, e, de outro, o cenário anti-moderno da crítica à ciência, à técnica, ao progresso que atualmente ocupa o mundo inteiro parece não estar em contradição à modernidade, mas antes representar seu desenvolvimento radical e consequente para além do projeto da sociedade industrial. (GOERGEN, 2010, p. 9)

En un texto muy actual que se denomina Las Cuestiones, Nicolás Casullo ${ }^{1}$ analiza en el primer capítulo la idea de "revolución" su relación constitutiva con las filosofías de la historia y lo que él denomina la relación binómica "historia y sentido de la historia" es decir, una relación que sustentó las nociones de progreso, emancipación, evolución, desenvolvimiento y que dejó de ser tenida en cuenta en la construcción de una buena parte de los imaginarios políticos y sociales contemporáneos, los motivos de ese abandono no son el tema central de la reflexión de Casullo, más bien le preocupa desentrañar ¿qué es lo que hay en el lugar en el que antes estaba la idea de "revolución”? y ¿cuáles son las consecuencias de ese espacio vacío? En ese sentido, entender la "revolución como pasado" cambia las coordenadas de análisis y abre la necesidad de redefinir lo que se entiende por crítica.

La noción de crítica ha estado unida al concepto de emancipación social, de revolución, de horizonte de transformación social. Ligada, consecuentemente, al destino de la filosofía de la historia. Era esa relación la que permitía explicar la educación como inherente al desenvolvimiento de la naturaleza humana y también permitía direccionar el proceso educativo.

${ }^{1}$ Sociólogo argentino, profesor de la Facultad de Ciencias Sociales de la Universidad de Buenos Aires, fallecido en el año 2008.

Filosofia e Educação [RFE] - Volume 8, Número 2 - Campinas, SP Junho-Setembro de 2016 - ISSN 1984-9605 - p. 79-96 
En ese sentido, la muy difundida idea de la crisis de la educación no debe buscarse en el interior de la escuela o del aula, la crisis de la educación se debe, en primer término, a la ruptura de esa relación. Casullo no se pregunta sobre el problema educacional sí, en cambio, se pregunta sobre las consecuencias que produce en el pensamiento crítico esa ruptura:

[...] se trata de interrogar el inmenso espacio deshabitado que se abrió en la inteligibilidad de las cosas, cuando este espacio sobre una ausencia no logró ni logra transformarse en una conciencia de época [...] Es decir la imposibilidad de pensar críticamente la cura política sobre el propio pensar lo nuevo. Un hueco no asumido como condición decisiva del estado de la crítica. (CASULLO, 2007, p. 12)

En esas palabras de Casullo se cifra el mayor desafío que enfrenta lo que podríamos denominar como "educación crítica", porque ese hueco que dejó la idea de emancipación social no ha sido cubierto. Asistimos, dice el autor,

a la dificultad de preguntarse, entonces, qué quedó de esa revolución que concluiría con una historia injusta a partir de una sociedad futura sin explotadores ni explotados. Preguntarse [...] donde antes había eso que pasó a haber? (CASULLO, 2007, p. 13)

La pregunta dirigida a las ciencias sociales y a la política resulta de vital importancia en el campo educacional, pues la creciente instrumentalización con la que es abordado, abandona esos temas sin haberlos agotado en su discusión, sin haberlos actualizado, es decir, dando por cerrado el ciclo de la posibilidad de emancipación social y con ello dando por cerrado el debate de lo que interpretamos como "pedagogía 
crítica", más bien, la crítica ahora está ligada al contexto, al presente, a la mirada auto-referencial, y en el caso educacional intra-institucional e intraescolar. Estudiar lo educacional es adentrarse en una problemática reducida al ámbito de la escuela, de las relaciones institucionales, de las prácticas didácticas, curriculares, estudiar el planeamiento, la evaluación, etc. ligados estrictamente al ámbito de emisión del discurso. En ese sentido, el espacio que ocupaba la noción de emancipación social fue ocupado por un enfoque crecientemente tecnocrático.

Entonces ¿cómo pensar la crítica sin referirla a la idea de emancipación social?

En el ámbito de la pedagogía, lo que se considera crítico se desdibuja y va paulatinamente perdiendo sus límites, su perfil, de tal modo que cuando se habla de pensamiento crítico no aparece un horizonte en el que podamos referenciarnos o, dicho de otro modo, muchas perspectivas teóricas, pueden entenderse o autodenominarse críticas, entre ellas, las que, explícitamente, aluden a un horizonte emancipatorio y también las que lo han abandonado.

En su texto Patologías de la razón, Honneth dice:

Desde hace años ya, en realidad desde el fin del marxismo como teoría autónoma, se discute desde las perspectivas más diversas qué posibilidad hay de encontrar en la actualidad un punto de vista adecuado para llevar a cabo una indagación crítica de las sociedades liberal-democráticas sin un préstamo de la filosofía de la historia.

[...] el problema central aquí es cómo describir un punto de vista desde el cual se pueda hacer una crítica teóricamente productiva de la sociedad y de sus prácticas institucionales. (HONNETH, 2009, p. 53)

Filosofia e Educação [RFE] - Volume 8, Número 2 - Campinas, SP Junho-Setembro de 2016 - ISSN 1984-9605 - p. 79-96 
En otros textos ya nos hemos referido a este tema, la imposibilidad de sostener la filosofía de la historia produce en el campo crítico un desamparo que abarca la noción de transformación social, de sentido de la historia, y de emancipación ${ }^{2}$. Este es el principal motivo por el cual la relación entre crítica y educación se torna tan problemática y también es el motivo por el cual precisa ser colocada una y otra vez en el panorama de la teoría de educación contemporánea.

En el escenario de lo que se denominan ciencias de la educación, la crítica está reducida a un simple rechazo de las prácticas educacionales que se observan cotidianamente, a un rechazo de las políticas públicas en educación, a una denuncia permanente dirigida al modo en que se desarrollan los sistemas educacionales. En suma, lo que en el campo pedagógico se denomina crítica es, esencialmente, la impugnación de las prácticas y las políticas que caracterizan a los sistemas educacionales actuales. La pregunta que se impone es ¿en nombre de qué se impugnan las prácticas educacionales o los discursos pedagógicos o las relaciones institucionales? Y ahí sobreviene el problema central en el que se debate el tema de la relación entre crítica, emancipación y educación en la actualidad.

En el breve texto que ya cité de Honneth, el autor alemán hace referencia a la consabida diferenciación entre dos formas de crítica: fuerte y débil, la forma de crítica fuerte sería aquella capaz de trascender el "horizonte de valores establecidos localmente apelando a principios morales externos, universalistas" lo que, según sus críticos, ${ }^{3}$ llevaría a una "perspectiva, demasiado distanciada como para que sus destinatarios puedan entenderla; y por eso es que siempre corre el riesgo de reivindicar un saber

${ }^{2}$ Ver SGRÓ, Margarita. Educação pós-filosofia da história: Racionalidade e emancipação. São Paulo: Cortez Editora, 2007.

${ }^{3}$ Honneth cita a Rorty y Walzer. Ver HONNETH, Axel. Patologías de la razón. Historia y actualidad de la Teoría Crítica. España: Katz, 2009.

Filosofia e Educação [RFE] - Volume 8, Número 2 - Campinas, SP Junho-Setembro de 2016 - ISSN 1984-9605 - p. 79-96 
especial elitista del que fácilmente se puede abusar con fines de manipulación." (HONNETH, 2009, p. 54)

Una crítica débil, en cambio, sería aquella que no trasciende los límites de la "cultura moral que prevalece en esa sociedad" de tal forma que "el crítico no estaría en condiciones de identificar como anomalía social algo que también los demás miembros de la sociedad puedan percibir potencialmente como injusto". (HONNETH, 2009, p. 54)

De tal modo que, como afirma Honneth, para los defensores de esta última posición:

[...] sólo una forma débil de crítica de la sociedad, ligada al contexto, constituye una empresa legítima desde el punto de vista político y filosófico (nosotros agregaríamos pedagógico), mientras que toda forma fuerte de crítica de la sociedad, que trascienda el contexto conlleva forzosamente los riesgos del paternalismo o incluso del despotismo. (HONNETH, 2009, p. 54)

Esa doble perspectiva de análisis permite colocar el problema de la educación contemporánea y la debilidad de la crítica que no puede trascender su propio marco referencial, resultando con ello improductiva para orientar la acción. En esa colocación parece estar la problemática fundamental de la "pedagogía crítica", el callejón sin salida en el que se encuentra, si no consigue excederse a sí misma. Dicho de otro modo, sortear los límites del presente sería para los que asumen la postura de una crítica débil entrar en un terreno de arenas movedizas en el que la emancipación social, la historia y sus sentidos pertenecen definitivamente al pasado. En su lugar quedó un vacío que no se puede llenar simplemente 
ignorando los fracasos de los procesos de transformación social que registra la historia reciente.

En ese sentido, la crítica "ligada al contexto" está impedida de pensar más allá de los límites impuestos por él, se ve impedida de pensar la relación educación-transformación social, e imposibilitada de pensar la contribución posible de lo educacional a un eventual proceso de emancipación social. Atados a un criterio de crítica débil los procesos educacionales y más aún los procesos de enseñanza y aprendizaje deben ceñirse al propósito de la adaptación social, utilidad en la formación para el empleo o ciudadanía adaptativa, todos ellos, procesos destinados a la reproducción social. Sin embargo, la educación también debería tener un papel destacado en la innovación social y contribuir a marcar la orientación de los procesos de transformación social.

O que efetivamente necessitamos são novos recursos conceituais que, sem perder a relação com o antigo, nos permitam pensar e agir no contexto do novo e complexo que nos envolve. Somos hoje herdeiros de uma crítica cultural que não pode dar-se por satisfeita com o pessimismo que lhe é inerente, mas precisa pensar o novo, o não-ser, na certeza de que o mundo não se acaba porque há mudanças. Tratase, sobretudo, de pensar os sentidos humanos dos processos da modernização, em particular aqueles que agregaram enorme poder e centralidade ao progresso científico/tecnológico. (GOERGEN, 2010, p. 10)

Pensar y actuar en el contexto de lo nuevo y complejo de la contemporaneidad, he ahí el desafío de la teoría de educación. 
Nos hemos servido parcialmente de un análisis del concepto de crítica enmarcado en la "Teoría crítica de la sociedad" para comprender los problemas inherentes a la conceptualización de la crítica en las sociedades actuales y entender la necesidad de repensarla, sin embargo por mucho que la colocación sobre ella ilumine las características elementales del tema en la actualidad siempre resulta un reto pensarla en el campo educacional, porque el sentido de la tendencia a la emancipación social con el que hemos definido la pedagogía crítica precisa sustentarse en esta mirada, buscar fundamento en ella pero no puede resolverse en ella, la dimensión práctica, propia de los procesos de enseñanza y aprendizaje, que constituyen la pedagogía no pueden contentarse con esta formulación. Por eso el intento de buscar una fundamentación para la teoría crítica de educación no puede, simplemente, quedar formulado como un problema que no se proponga avanzar en especificaciones relativas a la configuración de los procesos de enseñanza y aprendizaje, de los contenidos de la enseñanza, de las metodologías de trabajo para la formación de ciudadanos. En esa dirección ensayaremos algunas respuestas de lo que constituiría una "pedagogía crítica".

Decíamos que una pedagogía crítica no debe abandonar su pretensión de emancipación social como fin, pues en esa pretensión reside su legitimidad, pero tal vez debamos avanzar más en su definición.

Fazer perguntas num contexto de respostas prontas vindas em grande medida inclusive do contexto europeu foi a tarefa que se pôs Paulo Freire. Na sua visão a tarefa da teoria da educação é tornar transparentes para os próprios atores os paradigmas teóricos que veladamente comandam seu agir. Cabe à teoria esclarecer o homem sobre o pensamento, sobre os sentidos de seu presente e futuro, sobre

Filosofia e Educação [RFE] - Volume 8, Número 2 - Campinas, SP Junho-Setembro de 2016 - ISSN 1984-9605 - p. 79-96 
o sentido de sua relação com o outro e com a natureza. Teoria da educação significa autotematização do pensamento pedagógico com o objetivo de desvelar os pressupostos que orientam suas posturas e respostas. Significa distanciar-se de si mesmo, tematizar os próprios pressupostos e deixar-se afetar de forma dialógica por concepções e posicionamentos diferentes dos próprios. Olhar para si mesmo, desconfiar das próprias convicções e tomar consciência das posições dos outros não visa apenas o aperfeiçoamento do real pelo uso de estratégias racionais objetivas, mas a crítica radical das situações dadas de injustiça e desigualdade e a conquista de novo patamar político e ético. (GOERGEN, 2010, p.13)

En los manuales de Pedagogía o de teoría de la educación se denominan "Pedagogías críticas" a las interpretaciones teóricas cuya raíz se encuentra en el Marxismo y dentro de ese amplio espectro, con sus matices, también pueden considerarse las Pedagogías latinoamericanas de los años '60 y '70, a su vez cada una de estas opciones contienen varias líneas cuyos desarrollos parten de una caracterización similar de la sociedad y de sus relaciones económicas y de poder. Esa breve definición es hoy insuficiente aunque no inexacta, podríamos decir que llamamos educación crítica a una educación que se coloca como actor social en la reproducción y en la innovación de la sociedad, capaz de transformarse al paso que colabora con la transformación de la sociedad, capaz de dar cuenta de los problemas de su tiempo, no simplemente elaborando una descripción o un diagnóstico, sino esencialmente buscando en el tiempo presente las tendencias a la emancipación, pensando en perspectiva los tiempos que vendrán, su complejidad y su orientación democrática. Podríamos decir que lo que las miradas críticas tienen de común es la tendencia a la emancipación social y a la crítica. Definir lo que sea la emancipación social en el tiempo presente, 
sin duda está estrechamente unido a la lucha contra la desigualdad, esencialmente en nuestros países y sobre todo en los procesos políticos que se instalaron en los últimos 10 años. Entendemos por desigualdad la que deriva de la extremadamente inequitativa distribución de la riqueza pero también y esencialmente la que deriva de la falta de reconocimiento por la diversidad, cultural, sexual, religiosa, etc. en fin, de la falta de respeto por las opciones de los "otros".

Sin duda, la educación crítica está unida también, a una metodología dialógica, lo cual trae aparejada toda una discusión en sí misma que excede el límite de lo metodológico para entrar en el espacio del reconocimiento del otro, de sus temas, de sus problemas de sus interpretaciones. La pedagogía freireana desarrolló este tema con enorme amplitud porque en esa metodología dialógica se imprime una perspectiva ético-política. No desarrollaremos en este espacio lo referente a la Pedagogía de la liberación, sin embargo es necesario mencionarla cuando se piensa la dimensión práctica y las metodologías más adecuadas para la formación democrática.

Qué temas debe abordar una pedagogía crítica? Son varios y cambiantes acordes a la dinámica de los tiempos históricos pero en la actualidad, la efectivización de los derechos humanos que pasa cada vez más por derechos a una vida digna, salud, educación, vivienda, diversidad cultural, respeto por las diferencias y lucha contra la xenofobia, entre otros. La ecología y el cuidado del planeta, el desenvolvimiento sustentable, cuestiones de género y derechos de las minorías étnicas, religiosas, sexuales, la formación para la recepción crítica de los medios de comunicación hacen a la formación de un ciudadano del siglo XXI, responsable y solidario. Si bien estos temas no agotan, ni lo pretenden, el amplio escenario de lo que debe ser sistemáticamente abordado en el espacio escolar, son, por lo menos, algunos de los temas que no deberían ignorarse.

Filosofia e Educação [RFE] - Volume 8, Número 2 - Campinas, SP Junho-Setembro de 2016 - ISSN 1984-9605 - p. 79-96 
A toda esta problemática debe agregarse la cuestión de la formación de los profesores que no sólo debe enfocarse en una formación científica sólida que es imprescindible, sino en el urgente tema de la formación moral. Ahora bien, estas respuestas son necesariamente provisorias pero todas ellas exceden el estrecho marco de una perspectiva escolar o áulica, por ello, hemos intentado mostrar que para definir una educación crítica debemos recurrir a conceptos propios de miradas teóricas sobre el hecho educacional que nos permitan comprenderlo en un contexto que lo excede en gran medida, es decir, el hecho educacional puede describirse desde una perspectiva áulica pero sus alcances y su finalidad exceden al aula, a las relaciones que en ella se establezcan y a los resultados que cualitativamente se coloquen como objetivos propios del proceso de enseñanza y aprendizaje. En ese sentido el recorte del problema educacional para su estudio o para su proyección teórica ya implica una reducción del problema que termina modificándolo.

Por el contrario, lo que le permitiría a la educación ser una instancia de emancipación social y de autonomía del sujeto, es la posibilidad de pensar un horizonte futuro que, aunque anclado en el presente, no ha sido aún realizado. Releer el presente y reinventar las categorías que nos permitan ver las tendencias a la emancipación es lo que, en líneas generales, denominamos una teoría crítica de educación. 


\section{Referencias}

CASULLO, Nicolás. Las cuestiones. Buenos Aires: Fondo de Cultura Económica. 2007.

GOERGEN, Pedro. Pós-modernidade, ética e educação. Campinas, SP: Autores Associados. 2001.

Ação comunicativa, democracia e educação. In: SGRÓ, Margarita (org.). Teoría crítica de la sociedad, educación, democracia y ciudadanía. Tandil: Editora UNICEN, 2008.

Sociedade complexa, iluminismo pedagógico e pedagogia latino-americana. Tandil.

Inédito. 2010.

HONNETH, A. Patologías de la Razón. Historia y actualidad de la Teoría Crítica.

Trad. Griselda Mársico. Buenos Aires: Katz Editores, 2009. 